\title{
Electrochemical studies of ropinirole, an anti-Parkinson's disease drug
}

\author{
BILJANA NIGOVIĆ*, SANDRA JURIĆ, ANA MORNAR and INES MALENICA \\ Faculty of Pharmacy and Biochemistry, University of Zagreb, A Kovacica 1, 10000 Zagreb, Croatia \\ e-mail: bnigovic@pharma.hr
}

MS received 7 February 2012; revised 11 July 2012; accepted 27 September 2012

\begin{abstract}
The oxidation behaviour of a potent anti-Parkinson's disease drug ropinirole hydrochloride was investigated over a wide $\mathrm{pH}$ range in aqueous solution at glassy carbon electrode using cyclic and square-wave voltammetry. The oxidation of drug is a $\mathrm{pH}$ dependent irreversible process and occurs in two steps. The mechanism of the oxidation process has been discussed. Using the sharp oxidation response in $0.1 \mathrm{M}$ sulphuric acid at a potential of $+1.27 \mathrm{~V}$ attributed to the oxidation of indol-2-one ring in drug molecule, rapid electroanalytical methods for the determination of ropinirole by pulse voltammetric techniques were developed and validated. The proposed voltammetric methods were applied to direct quantification of ropinirole in film-coated tablets, with results in close agreement (at 95\% confidence level) with those obtained using a comparative HPLC method.
\end{abstract}

Keywords. Ropinirole hydrochloride; electrochemical oxidation; voltammetry; glassy carbon electrode; pharmaceutical analysis.

\section{Introduction}

Ropinirole hydrochloride (ROP) is a new dopamine nonergoline agonist recently introduced into Parkinson's disease therapy (scheme 1$).{ }^{1}$ Parkinson's disease is a progressive, neurodegenerative disorder primarily affecting dopaminergic neuronal systems, with impaired motor function as a consequence. ROP is generally well tolerated, and it can be used alone or in combination with levodopa. ${ }^{2}$ Moreover, ROP is efficacious in the treatment of more advanced Parkinson's disease in patients experiencing motor complications after long term levodopa use. It can also be used to treat restless legs syndrome. ${ }^{3}$

Electrochemistry is fast growing area with a number of possible applications in the pharmaceutical field. ${ }^{4}$ It can be used at the early stage of drug research for electroorganic synthesis of pharmacologically interesting molecules and screening the activity of newly synthesized molecules due to correlations noted between the redox potential and the pharmacological activity. Electron transfer reactions play important role in understanding the mechanism of action of various drugs and can serve as a useful tool in the design of more active and safer pharmaceuticals. The knowlage of redox properties of drugs can give insights into their metabolic fate or in vivo redox processes. ${ }^{5}$ In drug research, electrochemical techniques have application to drug-protein

*For correspondence and drug-DNA binding studies giving results useful in drug bioavailability and toxicity tests. Electrochemical methods cover a large domain of investigation in drug analysis ranging from the assay of drugs in bulk form, pharmaceutical formulations and drug therapy monitoring in biological fluids. Although the relevance of electrochemical techniques is discussed in the literature ${ }^{6}$ there has been no study published on the electrochemical redox properties of ROP and no attempt has been made till date to determine it by voltammetric methods. Electrochemical techniques offer an alternative approach to drug quantification, but they can also find the optimal solutions for many problems in pharmaceutical analysis. Apart from relatively simple and inexpensive instrumentation as well as short analysis time, the electroanalytical methods have proved to be sensitive and reliable for measuring in untreated samples. ${ }^{7-9}$

Literature review revealed that very few analytical methods were developed for the determination of ROP. Liquid chromatography-mass spectrometry methods were reported for quantification of ROP in biological fluids. ${ }^{10,11}$ The HPLC methods with UV detection were used for drug impurity profiling ${ }^{12}$ and stability-indicating assays. ${ }^{13,14}$ Separation and quantification of ROP and its impurities have been attempted using capillary liquid chromatography ${ }^{15}$ and capillary zone electrophoresis. ${ }^{16}$ Spectrophotometry, ${ }^{17}$ spectrofluorimetry ${ }^{18}$ and ultra-performance liquid chromatohraphy ${ }^{19}$ were also proposed for the compound 


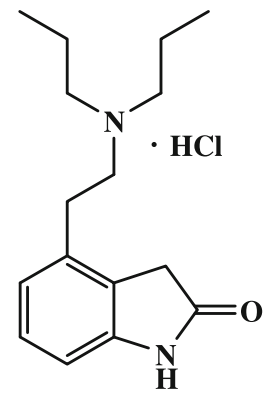

Scheme 1. Structure of ropinirole hydrochloride (ROP).

quantification. The official method for quantification of ROP as a raw material has not yet been approved in European Pharmacopoeia. ${ }^{20}$ Very little information about the analysis of ROP in pharmaceutical formulations is available in the literature. The assay for the determination of ropinirole in tablet by HPLC technique was published, ${ }^{21}$ however simpler alternative methods for the estimation of drug content in bulk and pharmaceutical dosage forms are highly desirable. Commonly used separation techniques are superior when the drug has to be determined in the presence of its metabolites or process impurities and degradation products of the drug substance. But it may be questionable if chromatographic method should present the fastest procedure for determination of the active ingredient in pharmaceutical product with suitable accuracy.

The aim of the present study was to examine the oxidative properties of ROP over a wide $\mathrm{pH}$ range in aqueous solution at a glassy carbon electrode using cyclic voltammetry (CV) and square-wave voltammetry (SWV). Rapid and simple electroanalytical methods were developed for the direct determination of ROP in bulk form and its film-coated tablets using pulse voltammetric techniques. The HPLC was chosen as the comparative method for evaluating the proposed new procedures.

\section{Experimental}

\subsection{Instrumentation}

The cyclic, differential pulse and square-wave voltammetric experiments at a stationary electrode were performed using a $\mu$-Autolab potentiostat (Eco Chemie, Utrecht, The Netherlands) controlled by GPES 4.9 software. A three-electrode system incorporating a glassy carbon working electrode (GCE, $3 \mathrm{~mm}$ diameter, Metrohm, Switzerland), $\mathrm{Ag} / \mathrm{AgCl}$ ( $\mathrm{KCl} 3 \mathrm{M}$, Metrohm) reference electrode and a platinum counter electrode was used. Before each experiment, the GCE was polished thoroughly with aqueous slurry of $0.05 \mu \mathrm{m}$ alumina powder on a polishing pad, rinsed thoroughly with deionized water and ultrasonicated for $30 \mathrm{~s}$. All measurements were performed at room temperature $(23 \pm$ $2^{\circ} \mathrm{C}$ ) in a $20 \mathrm{~mL}$ electrochemical cell.

$\mathrm{CV}$ was carried out from 0 to $1.5 \mathrm{~V}$ with the scan rate varying from 10 to $500 \mathrm{mV} \mathrm{s}^{-1}$. Operating instrumental conditions for SWV were: pulse amplitude of $50 \mathrm{mV}$, frequency of $120 \mathrm{~Hz}$ and scan increment of $10 \mathrm{mV}$. In differential pulse voltammetry (DPV) the following parameters were employed: pulse amplitude of $50 \mathrm{mV}$, pulse width of $50 \mathrm{~ms}$ and scan rate of $25 \mathrm{mV} \mathrm{s}^{-1}$. The voltammograms were recorded in the SWV and DPV modes by applying positive-going potential scan from 0.5 to $1.5 \mathrm{~V}$.

For the comparison study, HPLC experiments were carried out using an Agilent 1100 Series LC system (Agilent Technologies, Waldbronn, Germany) consisting of a vacuum degassing unit, a quaternary pump, a standard automatic sample injector, a column oven and a diode array detector. A chromatographic column XBridge C18, $3.0 \times 50 \mathrm{~mm}$, particle size $2.5 \mu \mathrm{m}$ (Waters, Miliford, Mass, USA) was used in quantification of ROP. The mobile phase consisted of acetonitrile, ultra-pure water and ammonium hydroxide $(25 \%)$ at the ratio of 400:600:0.04 (v/v/v). The mobile phase mixtures were filtered through cellulose nitrate filter $(0.45 \mu \mathrm{m}$, Sartorius, Goettingen, Germany). The HPLC analyses were carried out at constant temperature $\left(25^{\circ} \mathrm{C}\right)$ with a flow-rate of $1 \mathrm{~mL} / \mathrm{min}$ under isocratic conditions. For chromatographic analysis, standard solutions of ROP in mobile phase were filtered through a $0.2 \mu \mathrm{m}$ Acrodisc GHP filters (Gelman, Ann Arbor, USA). The $5 \mu \mathrm{L}$ aliquots were injected onto the HPLC system for analysis. The DAD detector recorded UV spectra in the range from 190 to $400 \mathrm{~nm}$ and chromatogram was obtained at $250 \mathrm{~nm}$. All chromatographic data acquisition and processing was performed using ChemStation software (Agilent Technologies, Waldbronn, Germany).

\subsection{Reagents and solutions}

Ropinirole hydrochloride, kindly donated by Pliva (Zagreb, Croatia), was used without any purification. Requip $\AA$ film-coated tablets (GlaxoSmithKline, London, United Kingdom) containing $2.28 \mathrm{mg}$ of ROP equivalent to $2 \mathrm{mg}$ ropinirole, were supplied from local pharmacy. All chemicals for preparation of supporting electrolytes were of analytical grade quality. Ultra-pure water used for preparation of stock and buffer solutions 
was obtained by a Milli-Q system (Millipore, Bradford, USA).

Stock solution for voltammetric measurements was prepared in ultra-pure water at a concentration of $1 \times$ $10^{-3} \mathrm{M}$ and stored at $4^{\circ} \mathrm{C}$ in a refrigerator. Standard solutions were prepared daily by diluting the stock solution with the selected supporting electrolyte just before use. As supporting electrolytes, $0.1 \mathrm{M}$ sulphuric acid, $0.04 \mathrm{M}$ Britton-Robinson buffer solutions $(\mathrm{pH} 2.0$ 12.0 ) and $0.2 \mathrm{M}$ acetate buffer ( $\mathrm{pH} 3.7-5.0)$ were used.

Ten film-coated tablets were weighted and crushed to a fine powder. An adequate amount of prepared powder, equivalent to a stock solution of concentration $2 \times 10^{-4} \mathrm{M}$, was weighed, transferred into a $50 \mathrm{~mL}$ volumetric flask and dispersed in purified water. The mixture was sonicated for $10 \mathrm{~min}$ to provide complete dissolution. The non-disolved excipients were waited to precipitate. The sample from the clear supernatant liquor was withdrawn and a series of dilutions was made with supporting electrolyte. For chromatographic analysis, the appropriate tablet solutions were prepared in mobile phase and filtered through a $0.2 \mu \mathrm{m}$ Acrodisc GHP filters. The nominal content of tablet amounts were calculated from the corresponding regression equations of previously plotted calibration plots. In order to clarify whether the excipients show any interference with the analysis, known amounts of the ROP were added to the pre-analysed solutions of tablet dosage forms. The recovery results were calculated using the related calibration equation after five repeated experiments.

\section{Results and discussion}

\subsection{Electrochemical oxidation of ROP}

Electrochemical data concerning the redox mechanism of ROP or its electroanalytical determination have not yet been reported in the literature. Therefore, in the first step ROP was subjected to CV studies with the aim of a detailed characterization of its electrochemical oxidation behaviour on the GCE. The cyclic voltammograms of $1 \times 10^{-4} \mathrm{M}$ ROP solution in $0.1 \mathrm{M}$ sulphuric acid, at a scan rate $\mathrm{v}=100 \mathrm{mV} \mathrm{s}^{-1}$, show one well-defined anodic peak $\left(\mathrm{Ox}_{1}\right)$ that occur at $+1.27 \mathrm{~V}$ (figure 1). By reversing the potential scan direction at $+1.5 \mathrm{~V}$, no reduction signal corresponding to the anodic response was observed, showing that the oxidation of drug molecule is an irreversible process. However, in the reverse scan a small cathodic peak $\left(\mathrm{Rd}_{1}{ }^{\prime}\right)$ appeared at $+0.56 \mathrm{~V}$. On the second positive steep, a new small anodic peak $\left(\mathrm{Ox}_{1}{ }^{\prime}\right)$ was observed at potential less positive than that of ROP. This anodic peak only appeared

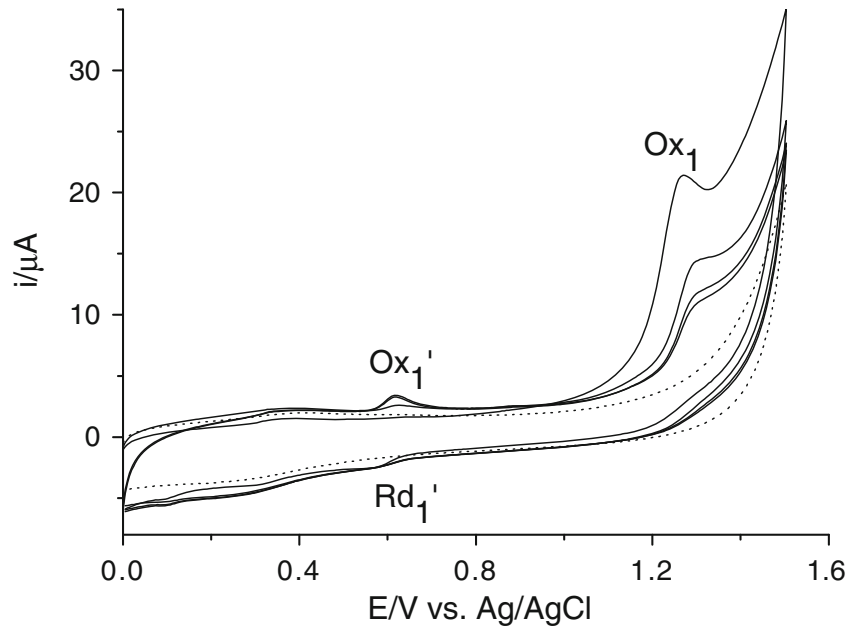

Figure 1. Successive cyclic voltammograms of $1 \times$ $10^{-4} \mathrm{M}$ ROP obtained at GCE in $0.1 \mathrm{M}$ sulphuric acid solution together with corresponding background recordings at a scan rate of $100 \mathrm{mV} \mathrm{s}^{-1}$.

after the initial oxidation of drug molecule and continued cycling of ROP confirmed the appearance of new reversible redox couple $\left(\mathrm{Ox}_{1}{ }^{\prime} / \mathrm{Rd}_{1}{ }^{\prime}\right)$ located at considerably lower positive potential region than the parent compound. Therefore, it suggests that some chemical follow-up reaction has occurred at the initial charge transfer giving rise to a product more readily oxidized than drug molecule.

The successive square-wave voltammograms showed that the amplitude of new peak $\mathrm{Ox}_{1}{ }^{\prime}$ at $+0.62 \mathrm{~V}$ augmented as the number of scans increase (figure 2). Therefore, this peak corresponds to the oxidation of the

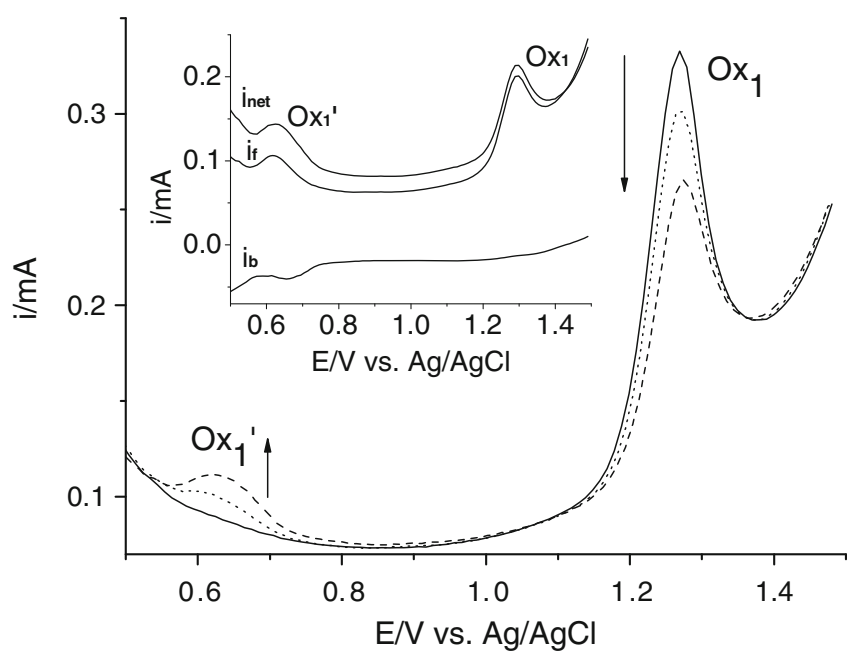

Figure 2. Successive square-wave voltammograms (SWV) of $1 \times 10^{-4} \mathrm{M}$ ROP obtained at GCE in $0.1 \mathrm{M}$ sulphuric acid. Inset shows the net current $\left(\mathrm{I}_{\text {net }}\right)$ together with its forward $\left(\mathrm{I}_{\mathrm{f}}\right)$ and backward $\left(\mathrm{I}_{\mathrm{b}}\right)$ components of the SWV response after fourth scan. SWV settings: amplitude of $50 \mathrm{mV}$, potential step of $10 \mathrm{mV}$ and frequency of $120 \mathrm{~Hz}$. 
product of chemical followed-up reaction of ROP oxidation, which is adsorbed on the electrode surface. At the same time, the oxidation current of ROP decreased gradually with the number of successive scans due to the decrease of available electrode surface area. Furthermore, the adsorption of the product at the GCE surface was confirmed when, after several SWV scans recorded in the solution of drug, the electrode was washed with deionized water and then transferred to the supporting electrolyte solution. The square-wave voltammogram obtained in these conditions shows only the oxidation of the product peak $\mathrm{Ox}_{1}$ '.

SWV is a technique that enables simultaneous inspection of both reduction and oxidation processes and hence provides an insight into the mechanism of the electrode reaction. The irreversibility of drug oxidation process in acidic aqueous medium and reversibility of peak at $+0.62 \mathrm{~V}$ were corroborated by plotting the forward and backward components of the SWV net current (inset of figure 2). Such voltammetric behaviour implies that the electrode oxidation of ROP in $0.1 \mathrm{M}$ sulphuric acid proceeds according to the EC (electron-transferchemical) mechanism where the oxidation product is transformed further to the electrochemically active product by a fast chemical reaction.

The influence of the scan rate on the electrochemical response of drug molecule in $0.1 \mathrm{M}$ sulphuric acid was investigated by $\mathrm{CV}$. The effects of the potential scan rate in the range $10-500 \mathrm{mV} \mathrm{s}^{-1}$ on the peak potential and the peak current of $\mathrm{Ox}_{1}$ were evaluated. As to an irreversible electrode process, the oxidation peak potentials $\left(\mathrm{Ep}_{1}\right)$ shifted to more positive potentials as the scan rate increased. Based on this, the number of electrons involved in the oxidation of ROP can be evaluated. The dependence of the peak potential is linear with logarithm of the scan rate with a slope of $-24.7 \mathrm{mV}$ decade $^{-1}$, allowing the calculation of $\alpha n=$ 1.20. Using the value of the charge transfer coefficient $(0.62)$ obtained from the difference between the peak potential $\left(\mathrm{E}_{\mathrm{p}}\right)$ and the half wave potential $\left(\mathrm{E}_{\mathrm{p} / 2}\right)$ using the equation $\Delta \mathrm{E}_{\mathrm{p}}=\mathrm{E}_{\mathrm{p}}-\mathrm{E}_{\mathrm{p} / 2}=(47.7 / \alpha) \mathrm{mV}$, the number of electrons exchanged was calculated to be $n=1.94$. A plot of logarithm of peak current versus logarithm of scan rate gave a straight line log $i=$ $8.28 \times 10^{-4}+0.5821 \log \mathrm{v}(\mathrm{r}=0.998)$ with a slope very close to the theoretical value of 0.5 , an expected value for an ideal reaction of solution species. In additional, the linear relationship existing between peak current and square root of the scan rate yielded a straight line, indicating that the electrooxidation of ROP in $0.1 \mathrm{M}$ sulphuric acid is a diffusion-controlled process. In addition, the enhancement of the peak current of $\mathrm{Ox}_{1}$ was not observed at open circuit accumulation using
SWV. The adsorption of ROP on the electrode surface could not be used as an effective preconcentration step prior to voltammetric quantification of the drug due to surface-active properties of drug oxidation product.

Furthermore, the electrochemical behaviour of ROP was studied over a wide $\mathrm{pH}$ range (1.0-12.0) in buffered aqueous media. Various supporting electrolytes were investigated including Britton-Robinson buffer, acetate buffer and sulphuric acid. Due to the poorly resolved signals obtained by $\mathrm{CV}$ with an increase in $\mathrm{pH}$, the effect of $\mathrm{pH}$ on peak intensity and peak potential were studied also using SWV techniques. The voltammetric response of drug was strongly $\mathrm{pH}$ dependent. ROP was electrochemically oxidized at GCE in two steps over the $\mathrm{pH}$ range investigated. It exhibited only one anodic peak $\left(\mathrm{Ox}_{1}\right)$ at $\mathrm{pH}<4.0$, while two well-defined oxidation peaks were observed in supporting electrolytes over the $\mathrm{pH}$ range 5.0-11.0 (figure 3). A new anodic peak $\left(\mathrm{Ox}_{2}\right)$ appeared at a less-positive potential than that of $\mathrm{Ox}_{1} . \mathrm{CV}$ and SWV measurements showed an irreversible nature of both oxidation processes. At $\mathrm{pH}$ above 11.0, these two processes were fused into only one broad peak.

The peak potentials of both oxidation processes were shifted to the less-positive potential values by increasing the $\mathrm{pH}$. This implies that $\mathrm{H}^{+}$ions are involved in the oxidation of ROP molecule. The effect of $\mathrm{pH}$ on the peak potentials of both oxidation responses $\left(\mathrm{Ep}_{1}\right.$ and $\mathrm{Ep}_{2}$ ) of $1 \times 10^{-4} \mathrm{M}$ ROP solution is shown in figure 4. The plot of the peak potential of $\mathrm{Ox}_{1}$ versus $\mathrm{pH}$ showed one straight line between 2.0 and 11.0, which can be expresses by the following equations in BrittonRobinson buffer: $\mathrm{E}_{\mathrm{p}}(\mathrm{V})=1.51-0.061 \mathrm{pH}(\mathrm{r}=0.991)$

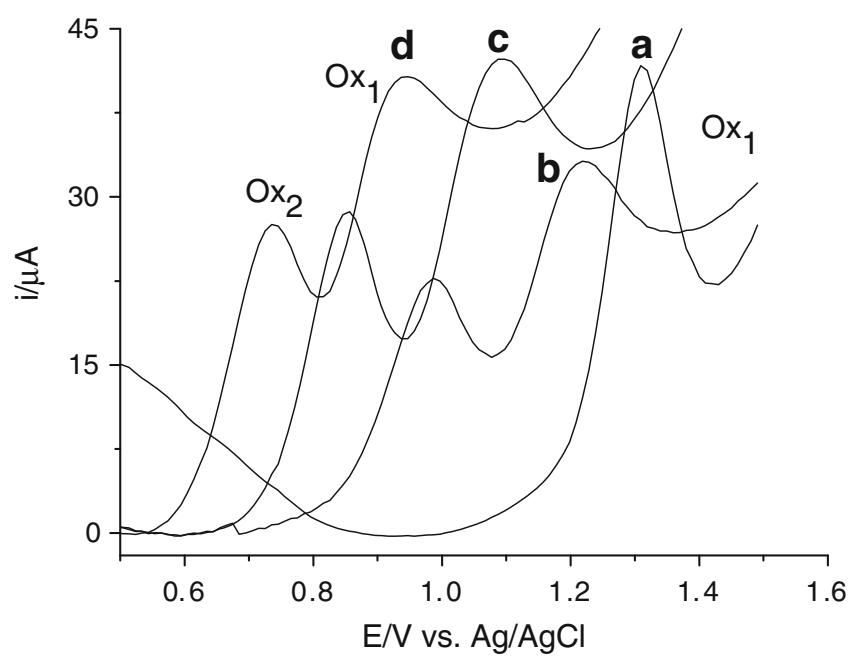

Figure 3. Square-wave voltammograms of ROP $(1 \times$ $10^{-4} \mathrm{M}$ ) obtained at the GCE in Britton-Robinson buffer solutions with pH 2 (a), 5 (b), 7 (c) and 9 (d); SWV settings same as in figure 2. 

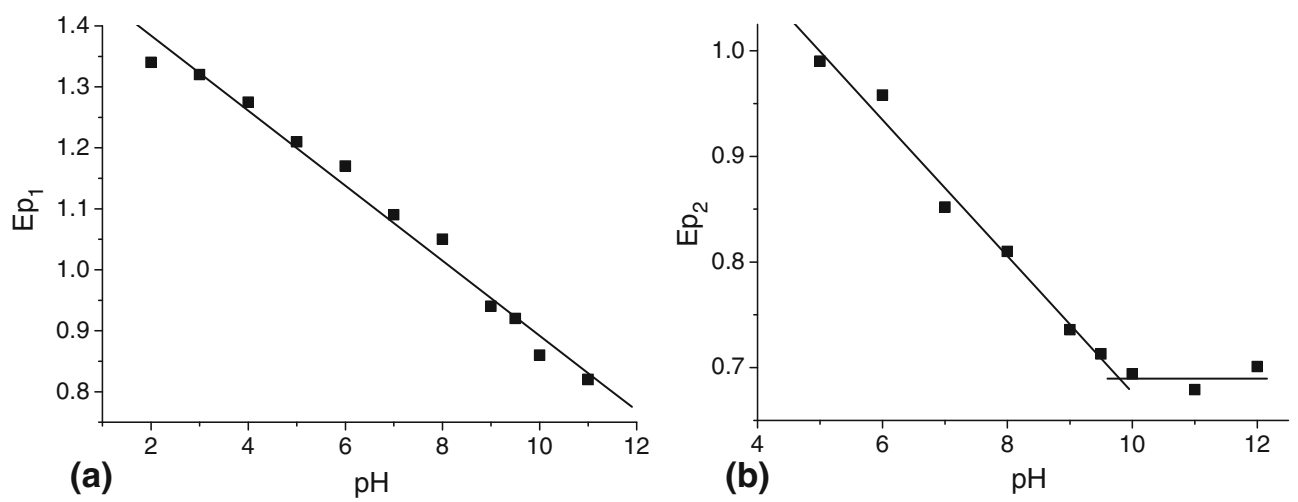

Figure 4. Effect of $\mathrm{pH}$ on square-wave voltammetric peak potentials for ROP solution $(1 \times$ $10^{-4} \mathrm{M}$ ) in Britton-Robinson buffer at GCE obtained with anodic peak $\mathrm{Ox}_{1}(\mathbf{a})$ and anodic peak $\mathrm{Ox}_{2}(\mathbf{b})$; SWV settings same as in figure 2.

for SWV. The linearity was observed for $\mathrm{Ox}_{2}$ in the $\mathrm{pH}$ range between 5.0 and 9.5, giving a negative slope of $64 \mathrm{mV}$ per $\mathrm{pH}$ unit $(\mathrm{r}=0.992)$. These slopes are close to the theoretical value of $59 \mathrm{mV} / \mathrm{pH}$, indicating participation of equal number of protons and electrons in the oxidation of ROP. Therefore, the oxidation of ROP in $0.1 \mathrm{M}$ sulphuric acid $\left(\mathrm{Ox}_{1}\right)$ belongs to a two-electron and two-proton process. The peak potential of $\mathrm{Ox}_{2}$ became practically $\mathrm{pH}$-independent above $\mathrm{pH}$ 10.0. The $\mathrm{pH}$-independent zone means that there is no proton transfer step before the electron-transfer rate-determining step. The $\mathrm{pH}$ dependence of the peak potentials and the intersection in $\mathrm{Ep}_{2}-\mathrm{pH}$ plot for $\mathrm{Ox}_{2}$ showed that the electroactive group that created this oxidation response has pKa value about 9.8. The intersection point observed in the plot is close to $\mathrm{pKa}$ value of ROP, which is reported to be 9.79 using capillary electrophoresis or 9.48 obtained by UV spectrophotometry, corresponding to loss of a proton from tertiary amine group. ${ }^{16,22}$ Therefore, the obtained results indicate that the electroactive group responsible for this oxidation process $\left(\mathrm{Ox}_{2}\right)$ observed in neutral and alkaline aqueous solutions is in acid-base equilibrium. The response $\mathrm{Ox}_{2}$ is probably caused by the oxidation of the nitrogen atom in the alkyl amine moiety. At much lower pHs $(<5.0)$, the oxidation generally became difficult due to the strong protonation of amine group of drug molecule. As the $\mathrm{pH}$ grew, the responses were enhanced due to the deprotonation prior to electron transfer.

Considering the molecular structure of ROP and the comparison with anodic voltammetric behaviour of some structurally related molecules with indol-2-one ring such as isatin and zisprasidone $e^{23,24}$ as well as the possibility of obtaining an oxidation signal in strong<smiles>CCC[NH+](CCC)CCc1cccc2c1CC(=O)N2CC(C)[C](C)[C](C)C</smiles><smiles>CCC[NH+](CCC)CCc1cccc2c1CC(=O)N2</smiles>

Scheme 2. Possible oxidation pathways of ropinirole hydrochloride at glassy carbon electrode. 
acidic media, the oxidation process $\mathrm{Ox}_{1}$ can be attributed to the oxidation of indol-2-one moiety. Therefore, the obtained results suggest that the oxidation process occurred firstly on indol-2-one ring, which is electroactive in both acidic and basic media, leading probably to hydroxylation of the benzene ring (scheme 2). In supporting electrolytes $\mathrm{pH}$ values above 5.0, ROP gave two separate oxidation steps. Taking into account the break point of $\mathrm{Ep}_{2}$ vs. $\mathrm{pH}$ plot for $\mathrm{Ox}_{2}$ and anodic voltammetric behaviour of some drugs which have tertiary amine group as only electroactive site on the molecule like doxepin, ${ }^{25}$ the second oxidation step $\left(\mathrm{Ox}_{2}\right)$ appeared in neutral and alkaline conditions could be located on the aliphatic nitrogen. After deprotonation, ROP lost an electron to form a cation radical which in subsequent step formed a quaternary Schiff base by losing a proton and an electron. Analysing the evolution of the SWV peak currents, it is possible to observe that this parameter shows dependence on the $\mathrm{pH}$ of the medium and supporting electrolyte composition. The peak $\mathrm{Ox}_{1}$ decreased gradually by increasing the $\mathrm{pH}$ value. The slowly decrease observed was accompanied by an appearance of second oxidation step $\mathrm{Ox}_{2}$ at less positive potentials. The second oxidation process became more pronounced as the $\mathrm{pH}$ decreased due to deprotonation of amine group, however, the voltammetric signals $\mathrm{Ox}_{1}$ and $\mathrm{Ox}_{2}$ were not well-resolved. On the other hand, the peak current of $\mathrm{Ox}_{1}$ was best developed in the form of a sharp peak and was easily measurable as a single response in strong acidic media. The anodic peak $\mathrm{Ox}_{1}$ reached the highest value in $0.1 \mathrm{M}$ sulphuric acid, which consequently was selected as the optimum supporting electrolytes for electroanalytical studies.

\subsection{Analytical determination of $R O P$}

On the basis of the electrochemical oxidation of ROP two different voltammetric methods using SWV and DPV techniques were developed for the quantitative determination of the drug. The anodic peak $\mathrm{Ox}_{1}$ was used for analytical studies and construction of calibration plot. The best single peak shapes as well as the peak current sensitivity were obtained in $0.1 \mathrm{M}$ sulphuric acid. Using the optimum instrumental conditions described in experimental section, the voltammograms for various concentration of ROP were recorded by applied techniques (figure 5). The linear calibration curves were obtained for ROP in the concentration range of $5 \times$ $10^{-7}-2 \times 10^{-5} \mathrm{M}$ for SWV and $1 \times 10^{-6}-2 \times 10^{-5} \mathrm{M}$ for DPV, as shown in the inset of figure 5. Above these concentration ranges, the loss of linearity was probably due to the adsorption of ROP on the electrode surface. The analytical characteristics of both methods
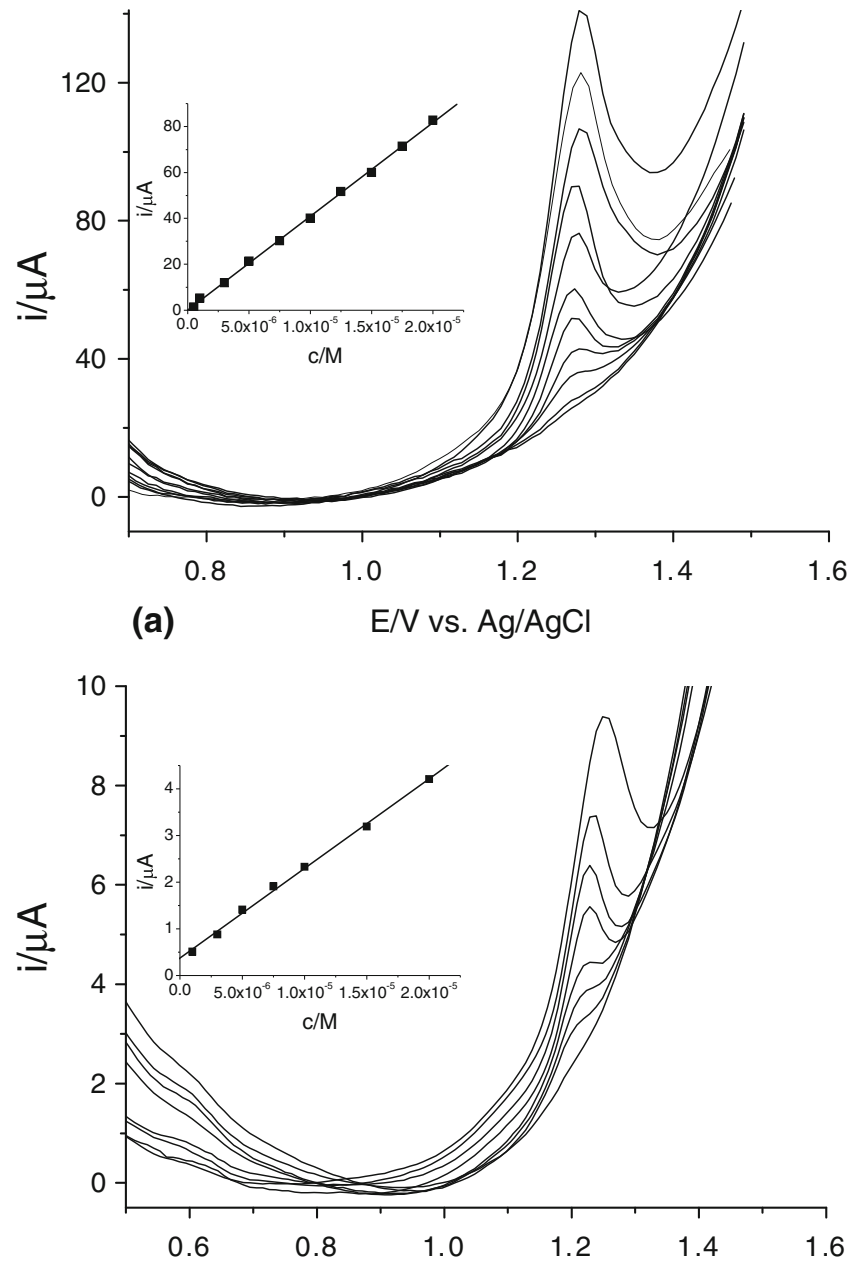

(b)

E/V vs. Ag/AgCl

Figure 5. SWV (a) and DPV (b) voltammograms of ROP for increasing drug concentrations at the GCE recorded in $0.1 \mathrm{M}$ sulphuric acid together with corresponding background recordings; SWV settings same as in figure 2. DPV settings: pulse amplitude $50 \mathrm{mV}$; pulse width $50 \mathrm{~ms}$; scan rate $25 \mathrm{mV} \mathrm{s}^{-1}$. Inset depicts a corresponding calibration plot for the quantification of ROP.

and related validation parameters are summarized in table 1 . The detection limit (LOD) and the quantification limit (LOQ) of both procedures were calculated from the calibration curves using the formula LOD = $3 s / m$ and $\mathrm{LOQ}=10 \mathrm{~s} / \mathrm{m}$, where $s$ is the standard deviation of the intercept and $m$ is the slope of the calibration curve. ${ }^{26}$ LOD and LOQ for newly developed procedures are also shown in table 1.

In comparison to DPV method developed, the advantages of SWV method are greater speed of analysis, reduced problems with blocking of the electrode surface and higher sensitivity. It should be mentioned once again that there is no article reporting on voltammetric method for the determination of ROP. Therefore, the analytical characteristics observed during validation 
Table 1. Analytical parameters for the calibration curves of ropinirole hydrochloride determination by SWV, DPV and HPLC methods.

\begin{tabular}{|c|c|c|c|}
\hline Parameter & SWV & DPV & HPLC \\
\hline Linearity range (M) & $5 \times 10^{-7}-2 \times 10^{-5}$ & $1 \times 10^{-6}-2 \times 10^{-5}$ & $2.5 \times 10^{-6}-2 \times 10^{-4}$ \\
\hline Slope $\left(\mu \mathrm{A} \mathrm{M} \mathrm{M}^{-1}\right)$ & $4.08 \times 10^{6}$ & $1.92 \times 10^{5}$ & $2.29 \times 10^{6}$ \\
\hline Intercept $(\mu \mathrm{A})$ & 0.04 & 0.38 & -0.37 \\
\hline SE of slope $\left(\mu \mathrm{A} \mathrm{M}^{-1}\right)$ & $4.44 \times 10^{4}$ & $4.54 \times 10^{3}$ & $4.91 \times 10^{3}$ \\
\hline SE of intercept $(\mu \mathrm{A})$ & 0.151 & 0.016 & 0.402 \\
\hline Correlation coefficient & 0.999 & 0.998 & 0.999 \\
\hline Limit of detection (M) & $1.1 \times 10^{-7}$ & $2.5 \times 10^{-7}$ & $5.3 \times 10^{-7}$ \\
\hline Limit of quantitation (M) & $3.7 \times 10^{-7}$ & $8.3 \times 10^{-7}$ & $1.8 \times 10^{-6}$ \\
\hline $\begin{array}{l}\text { Repeatability of peak } \\
\text { current/peak area (RSD\%) }\end{array}$ & 1.75 & 2.35 & 0.81 \\
\hline $\begin{array}{l}\text { Repeatability of peak } \\
\text { potential/retention time (RSD\%) }\end{array}$ & 0.67 & 0.61 & 0.57 \\
\hline $\begin{array}{l}\text { Reproducibility of peak } \\
\text { current/peak area (RSD\%) }\end{array}$ & 2.26 & 2.63 & 1.00 \\
\hline $\begin{array}{l}\text { Reproducibility of peak } \\
\text { potential/retention time (RSD\%) }\end{array}$ & 0.73 & 1.11 & 1.49 \\
\hline
\end{tabular}

of the proposed electrochemical methods were compared with those obtained by developed HPLC method for determination of ROP. As a part of HPLC validation procedure, system suitability parameters were checked by evaluating tailing factor (1.04) and theoretical plate number (2858). The developed HPLC method with UV-detection was validated according to standard procedure. ${ }^{27}$ The voltammetric methods exhibited lower linear range and moreover better sensitivity was obtained in the case of newly proposed SWV procedures. The proposed voltammetric methods are simpler, faster and cheaper. Compared with other earlier reported methods, the detection limits of ROP obtained by voltammetric methods are of the same order as for HPLC with UV detection ${ }^{21}$ and are better than that obtained in capillary electrophoresis ${ }^{16}$ and spectrophotometry, ${ }^{17}$ but are higher than LODs reported for chromatographic methods coupled to mass spectrometry ${ }^{10,11}$ and spectrofluorimetric method reported previously. ${ }^{18}$ However, spectrofluorimetric method additionally require derivatization of ROP with 4-chloro-7nitrobenzofurazan before detection step, while LC-MS analytical procedures demand expensive and sophisticated equipment that could not be available in many laboratories. The quantification limits are also comparable to some HPLC methods with UV detection, ${ }^{13,14}$ but the proposed electroanalytical methods offer several advantages over chromatographic techniques applied only to the quantification of drug, including short analysis time, simplicity of operation and lower running cost. Safe disposal or recycling of considerable amounts of expensive and toxic solvents used in the chromatographic analysis results in additional cost. The only disadvantage of the present procedures is that the renewal of the electrode surface was required after each experiment due to adsorption of the chemical product onto the electrode surface. However, stirring for $240 \mathrm{~s}$ eliminated the signal decrease in multi-scan voltammetric recordings. The responses were reproducible up to 10 repetitions (RSD of $3.5 \%$ ), and thereafter the signals started to decrease indicating the need for mechanical polishing of the electrode surface in order to obtain reproducible voltammetric response.

The stability of $5 \times 10^{-6} \mathrm{M}$ ROP standard solution was studied during a $72 \mathrm{~h}$ period at $4^{\circ} \mathrm{C}$ stored in a refrigerator by monitoring the drug concentration. The obtained results presented no significant differences in the peak currents and potentials among the measurements, with relative standard deviations of $2.1 \%$, indicating that the degradation of drug in $0.1 \mathrm{M}$ solution of sulphuric acid was negligible. To evaluate the intra-day and inter-day precision of the voltammetric response, analysis of standard ROP solution at concentration $1 \times$ $10^{-5} \mathrm{M}$ was studied by six replicate measurements on the same day and over three consecutive days by performing three measurements on each day using different standard solutions. Related parameters are given in table 1 . The obtained results indicate the good precision of the proposed procedures.

\subsection{Application to the pharmaceutical product}

In order to assess the applicability of the proposed methods, ROP was analysed in commercial film-coated tablets. On the basis of above results, new developed SWV and DPV methods were applied to the direct 
Table 2. Analysis of ropinirole hydrochloride in film-coated tablets by the proposed SWV, DPV and HPLC methods.

\begin{tabular}{lccr}
\hline Technique & SWV & DPV & HPLC \\
\hline Stated content $(\mathrm{mg})$ & 2.28 & 2.28 & 2.28 \\
Detected content $(\mathrm{mg})^{\mathrm{a}}$ & 2.31 & 2.27 & 2.35 \\
RSD \% & 1.91 & 2.44 & 0.49 \\
Bias \% & 1.32 & -0.44 & 3.07 \\
Added $10^{5} \mathrm{c}\left(\mathrm{mol} \mathrm{L}^{-1}\right)$ & 1.00 & 1.00 & 5.00 \\
Found $10^{5} \mathrm{c}\left(\mathrm{mol} \mathrm{L}^{-1}\right)^{\mathrm{a}}$ & 0.99 & 0.99 & 5.03 \\
Recovery \% & 99.6 & 99.4 & 100.6 \\
RSD \% & 1.42 & 1.59 & 0.28 \\
Bias \% & -0.36 & -0.56 & 0.60 \\
$F^{\mathrm{b}}$ & 1.95 & 2.19 & - \\
$t^{\mathrm{b}}$ & 0.06 & 0.08 & - \\
\hline
\end{tabular}

${ }^{a}$ Each value is the mean of five experiments

${ }^{\mathrm{b}}$ The theoretical values of $F$ and $t$-test at $95 \%$ confidence limit are 6.39 and 2.31, respectively

determination of ROP in its pharmaceutical product of current therapeutic use. Well-defined DPV and SWV peaks were obtained and no interferences were observed. The amount of active ingredient present in a tablet solution was calculated from the corresponding calibration equation/plot from the insets of figure 5 . Results summarized in the table 2 show that the content for assayed tablets was in agreement with the claimed amount. Application of the method to ROP determination in pharmaceutical formulations resulted in acceptable percent recoveries. The effect of excipients upon the voltammetric response for the examined drug was studied by adding known amount of drug standard to the formulation solution samples. The mean recovery of $100.4 \%$ indicated that excipients did not interfere with the assay, thus corroborating the suitability of newly introduced electroanalytical methods for this purpose. The proposed DPV and SWV methods proved to be sufficiently precise and accurate for reliable analysis of ROP in pharmaceutical preparations after a simple dilution step.

The film-coated tablets were also determined by the reverse-phase HPLC method. The results obtained with the proposed electroanalytical methods were compared with those obtained by HPLC method. Recovery experiments were also performed for HPLC method. Statistical comparison were performed on data obtained using both voltammetric and HPLC procedures. The results of the student $t$-test and variance ratio $F$-test show that there are no significant differences between the techniques with regard to accuracy and precision (table 2). However, the electroanalytical methods involve no sample preparation, do not require filtration, degassing and expensive solvents that are needed for HPLC procedure. The newly developed methods can be successfully used in controlling the quality of active pharmaceutical ingredient and dosage forms.

\section{Conclusions}

The electrochemical behaviour of ROP has been examined for the first time. The oxidation of ROP is a $\mathrm{pH}$ dependent, irreversible process and occurs in two steps. In the $\mathrm{pH}$ range 1.0-11.0, ROP oxidation involves transference of two electrons and two protons in indol2 -one ring. This voltammetric response is used for electroanalytical measurements of drug molecule. The electroactive group that created another oxidation response above $\mathrm{pH} 4$ has $\mathrm{pKa}$ value about 9.8, corresponding to tertiary amine group in drug molecule. Possible oxidation mechanism of ROP was discussed. New SWV and DPV methods for the electroanalytical determination of ROP were developed and validated. The voltammetric methods proposed were applied to direct quantification of ROP in film-coated tablets. The oxidative voltammetric behaviour of ROP investigated at the GCE could be used for the development of a reverse-phase HPLC procedure with electrochemical detection for trace determination of ROP. Due to noticeable shortage of methods described in the literature for ROP determination, the development of novel electrochemical sensors for its quantification will be of great importance. Improved electroanalytical performances of sensors require a particular attention owing to obtain surface stability, providing great possibilities for convenient repetitive voltammetric measurements without time-consuming polishing which is essential surface cleaning step in the case of GCE application. 


\section{Acknowledgements}

This work was supported through a grant (Investigation of new methods in analysis of drugs and bioactive substances) from the Ministry of Science, Education and Sports of the Republic of Croatia.

\section{References}

1. Matheson A J and Spencer C M 2000 Drugs 60115

2. Hubble J, Koller W C, Atchison P, Taylor A C, Citerone D R, Zussman B D, Friedman C J and Hawker N 2000 J. Clin. Pharmacol. 40641

3. Bogan R K 2008 Expert. Opin. Pharmacother. 9611

4. Nigović B 2009 In The analysis of pharmacologically active compounds and biomolecules in real samples, Ed. R Injac, Kerala: Transworld Research Network, pp 1-44

5. Uslu B and Ozkan S A 2007 Anal. Lett. 40817

6. Gupta V K, Jain R, Radhapyari K, Jadon N and Agarwal S 2011 Anal. Biochem. 408179

7. Nigović B and Hocevar S B 2011 Electrochim. Acta 58 523

8. Nigović B, Marušić M and Jurić S 2011 J. Electroanal. Chem. 66372

9. Nigović B and Spajić J 2011 Talanta 86393

10. Bhatt J, Jangid A, Shetty R, Shah B, Kambli S, Subbaiah G and Singh S 2006 J. Pharm. Biomed. Anal. 401202

11. Bharathi D V, Jagadeesh B, Kumar S S, Lakshmi R N, Hotha K K, Naidu A and Mullangi R 2009 Biomed. Chrom. 23557
12. Sahasrabuddhey B, Naudyal R, Acharya H, Khyade S, Luthra P K and Deshpande P B 2007 J. Pharm. Biomed. Anal. 431587

13. Azeem A, Iqbal Z, Ahmad F J, Khar R K and Talegaonkar S 2008 Acta Chromatogr. 2095

14. Parmar G, Sharma S, Singh K and Bansal G 2009 Chromatographia 69199

15. Coufal P, Stulik K, Claessens H A, Hardy M J and Webb M 1999 J. Chromatogr. B 732437

16. Coufal P, Stulik K, Claessens H A, Hardy M J and Webb M 1998 J. Chromatogr. B 720197

17. Onal A and Caglar S 2007 Chem. Pharm. Bull. 55629

18. Aydogmus Z 2008 Spectrochim. Acta Mol. Spectrosc. 7069

19. Krishnaiah C, Murthy M V, Reddy A R, Kumar R and Mukkanti K 2010 J. Chin. Chem. Soc. 57348

20. European Pharmacopoeia, 7th ed. 2012 (Strasbourg, Council of Europe)

21. Onal A 2006 Chromatographia 64459

22. Luzardo-Alvarez A, Delgado-Charro M B and BlancoMendez J 2001 Pharm. Res. 181714

23. Kul D, Gumustas M, Uslu B and Ozkan S A 2010 Talanta 82286

24. Diculescu V C, Kumbhat S and Oliveira-Brett A M 2006 Anal. Chim. Acta 575190

25. Xu X L, Huang F, Zhou G L, Zhang S and Kong J L 2010 Sensors 108398

26. Ermer J and Miller J H McB (Eds) 2005 Method validation in pharmaceutical analysis, Weinheim: Wiley-VCH Pub.

27. International Conference on Harmonization (ICH) 2005 Validation of analytical procedures: Text and methodology Q2 (R1) 